\title{
Microstructural and Fractographic Analysis on Samples of Fractured Bolts
}

\author{
A. C. Vidal* and A. R. Martins* \\ * Institute of Technology, Catholic University of Rio de Janeiro, Rua Marquês de São Vicente, 225, \\ Gávea, 22453-900, Rio de Janeiro, Brasil
}

Two fractured bolts from a tank ship were microscopically analyzed by means of optical and scanning electron microscopy in order to determine the actuating fracture micromechanisms. The material of both bolts was characterized by chemical analysis and the results showed that the composition of the samples corresponds to that specified for a SAE 4140 steel (Table 1).

Cross sections of each of the two samples were cut and prepared for optical micrography using standard metallography procedures (ASTM E3 Standard Methods). Metallographic examination of the samples showed a tempered martensitic structure, uniform over the entire cross section (Figure 1). Such microstructural aspects are expected for a 4140 steel quenched and tempered. There were not been observed along the analysed sections descarburized areas or the presence of discontinuities such as cracks and porosity.

The surfaces of fracture of both bolts were prepared to microscopic examination by scanning electron microscopy (SEM). In order to remove dirt and organic fluids from the fracture surfaces, they were cleaned by means of ultrassonic methods, washed with water and rinsed with acetone. Still after the cleaning procedure, the surface fracture presented several areas where mechanical and chemical damage could be visually observed. Mechanical damage may arise from several sources, including the striking of the fracture surface by other objects. This can occur during actual fracture in service or when removing or transporting a fractured part for analysis.

The fractographic analysis was carried out by means of scanning electron microscopy at different points of the samples. Figure 2 shows one of the fracture surfaces after cleaning where the two rectangles $A$ and $B$ indicate the studied areas.

Figure $3 \mathrm{a}$ is referent to area comprised in rectangle $A$ (Figure 2), where beach marks were visible during low magnification $(1,5 \mathrm{X}) \mathrm{SEM}$ examination. At high magnification, features typical of fatigue propagation were observed. Figures $3 b$ and $3 c$ refer to the analysed areas (Points 1 and 2, Figure 3a) with fatigue striations and the presence of secondary cracks. The area of the fracture surface identified by $B$ ( Figure 2 ) is presented in Figure $4 \mathrm{a}$ and Figures $4 \mathrm{~b}$ and $4 \mathrm{c}$ show the fractographies relative to the areas 1 and 2 (Figure 4a). The analysis of the area signed by 1 has indicated the predominance of fatigue striations and the presence of some intergranular facets as well as secondary cracks (Figure 4b). The final fracture occurred by the nucleation and coalescence of microvoids that formed as a result of particle-matrix decohesion or cracking of second-phase particles (Figure 4c). By the fractographic analysis, one can conclude that the fatigue crack initiations occurred in two different levels and the two resulting cracks joined by tearing. Final fracture occurred by ductile failure which ended on a plane inclined to the bolt axis. 


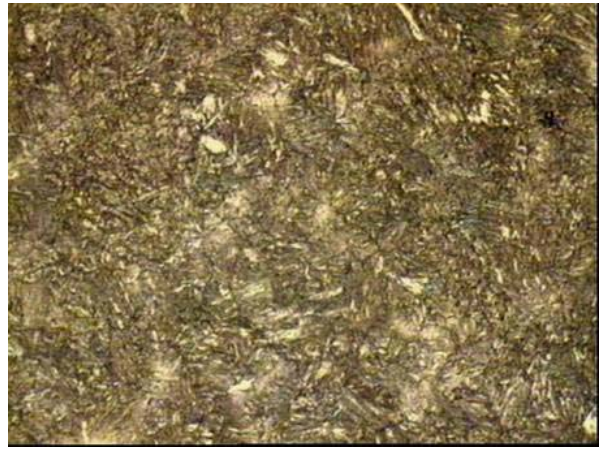

FIG. 1. Central area of the cross section, FIG. 2. which was characterized by a tempered martensitic structure. 250X.

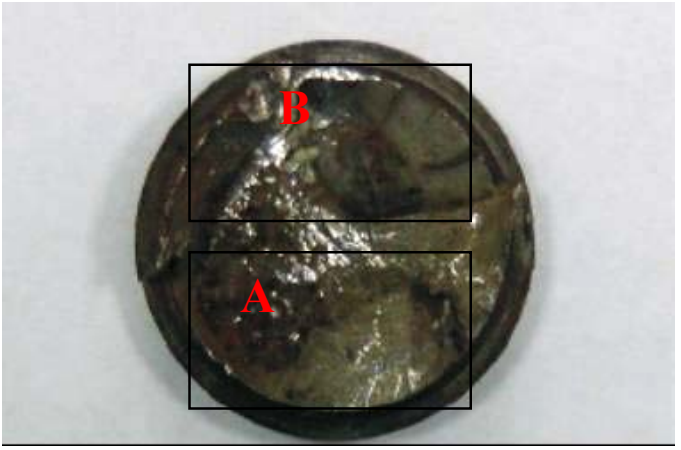
carried out in the areas comprised inside rectangles $\mathrm{A}$ and $\mathrm{B}$.
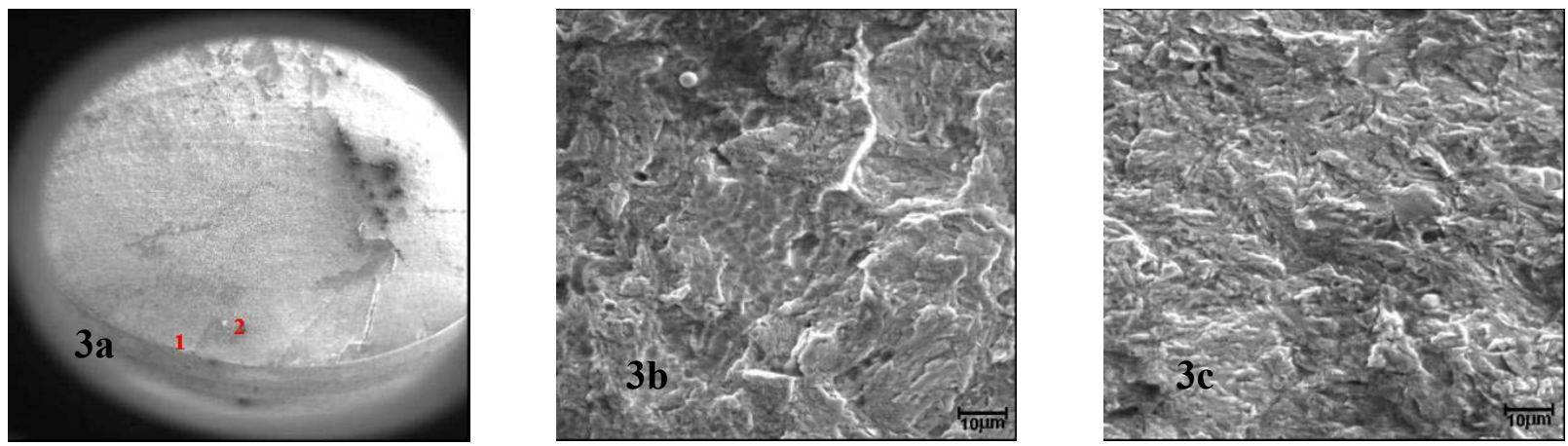

FIG. 3. (a) Area A shown in Figure 2. Presence of beach marks. (b) and (c) Points 1 and 2, Fig. 3a, fatigue striations are present characterizing the fatigue primary crack propagation.
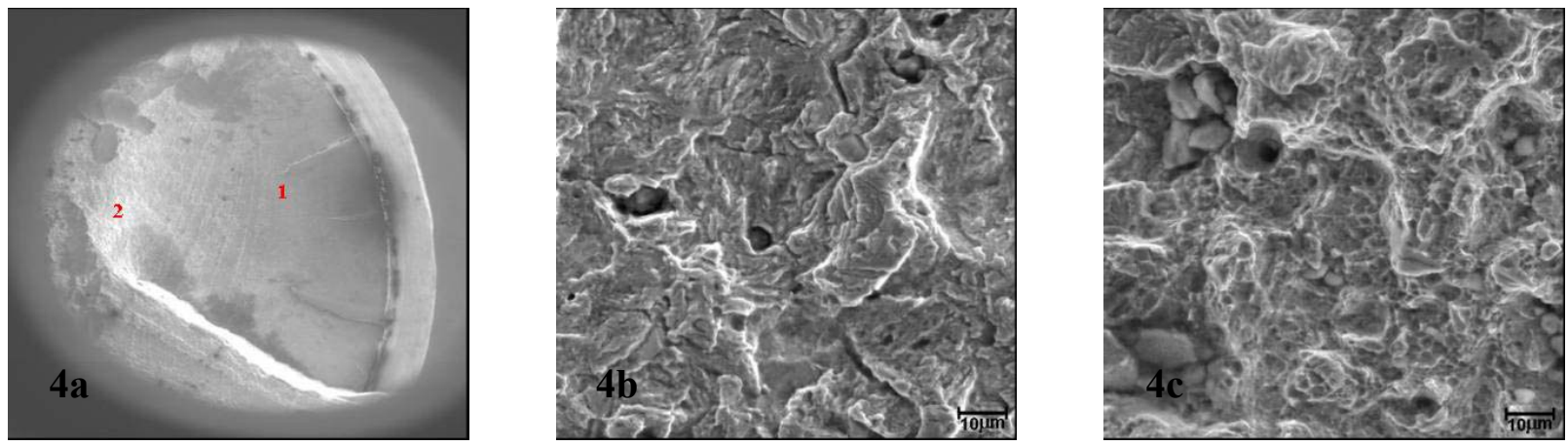

FIG. 4. (a) Area B shown in Figure 2. (b) Point 1, Fig 4a, predominance of fatigue striations and presence of some intergranular facets as well as secondary cracks. (c) Point 2, Fig. 4a, final fracture area. Thefracture occurred by the nucleation and coalescence of microvoids.

TABLE 1. Chemical composition in weight percent of the analysed samples.

\begin{tabular}{|c||c|c|c|c|c|c|c||c||c||c||c||}
\hline Bolt & C & Mn & Si & P & S & Cr & Ni & Mo & V & Cu & Al \\
\hline \hline 1 & 0,377 & 0,78 & 0,255 & 0,007 & 0,023 & 0,949 & 0,113 & 0,152 & 0,007 & 0,089 & 0,029 \\
\hline 2 & 0,379 & 0,80 & 0,222 & 0,007 & 0,024 & 0,951 & 0,115 & 0,158 & 0,007 & 0,091 & 0,028 \\
\hline
\end{tabular}

